\title{
VECTOR COMPRESSION METHOD TO CONVERT THE INCOMPLETE MATRIX OF PAIRWISE COMPARISONS IN THE ANALYTIC HIERARCHY PROCESS
}

\begin{abstract}
AHP is one of the most popular decision-making procedures due to its efficiency, flexibility and simplicity. Its main disadvantage is the inability to formalize incomplete or inaccurate comparisons of alternatives with each other. Missed assessments make it difficult to make decisions because most statistical methods do not apply to incomplete data sets. A popular classical algorithm for matrices of pairwise comparison processing cannot work with matrices containing predominantly zero components. The purpose of the work is to develop a method for expert assessments matrices processing in order to obtain weight coefficients (weights) of considered alternatives, which allow quantitative comparisons. The matrix of pairwise comparison is considered as a spanning graph in which compared alternatives are nodes and comparisons are edges. Here it is proposed a method of removing edges corresponding to the most controversial values, that is, a cycle break algorithm that causes the convert of the original graph into a spanning tree and allows uniquely comparing any two alternatives.
\end{abstract}

Keywords: decision making, analytic hierarchy process, incomplete matrices, spanning tree, vector compression method

\section{Introduction}

Decision-making procedures, in which experts are asked to choose the best variant (s) from an acceptable set, are often used in the very different areas to assess and define of priority of purposes, etc. However, there are some questions that are not currently fully answered: in particular, there is no method of working with incomplete matrices that allows not to restore the matrix to full; there are no methods that allow experts to determine the comparison ranges of pairwise assessments independently and there is no clear "stop criterion" if they want to achieve maximum consistency of assessments (each of existing methods has a tendency in "searching for consistency" to slide to "false weights" of analyzed alternatives). It is clear that the comparison of different alternatives according to their preference for decision-making tasks, in many cases, cannot be performed by a single criterion or by a single expert. As a result, in most decision-making tasks, there are procedures allowing to bring together views of different experts on their proposed alternatives. The multiplicative scale of AHP pairwise comparisons belongs to absolute scales (it is also the ratio scale). This is a subclass of interval scales in which there is a "zero point," which characterizes the absence of measured quality in the object. There is a need to use the views of different experts of different levels, as well as the second serious reason for the inconsistency of assessments - the inconsistency of views of the experts themselves intervenes in the assessments of the results. 
ISAHP Article: A Style Guide for Paper Proposals To Be Submitted to the International Symposium on the Analytic Hierarchy Process 2020, Web Conference.

\section{Literature Review}

Rubin, D. \& Little, R. (1987) and Millet, I. (1997) showed that as the result you can lose important conformities by dropping expert assessments, which often leads to false, but "coordinated assessments." The next stage in the development of AHP can be considered the methodology of setting an indistinct preference ratio, refusing to assess the proposed pair of alternatives Xu, Z.S. (2004), or even replacing contradictions with omissions. The principal applicability and effectiveness of one or another approach depends on the number of data drops and the reasons why they were generated Garcia-Laencina, P.J., SancoGomez, J.-L. \& Figueiras-Vidal, A.R. (2009). However, Carmone, F. J., Kara, Jr., A. \& Zanakis, S. H. (1997), a specific example shows that "randomly removing up to $50 \%$ of the comparisons gives good results without losing accuracy." In the presence of an incomplete matrix of pairwise comparisons, the authors proposed using methods that allow to predetermine the matrix to the full, as confirmed by Ebenbach, D.H. \& Moore, C.F. (2000). The system that helps to build indistinct preference relationships is proposed Alonso, S., Cabrerizo, F. J., Chiclana, F., Herrera, F. \& Herrera-Viedma, E. (2008). Group decision-making, description of procedures correcting the lack of knowledge of a particular expert, using information provided by other experts, together with some aggregation procedures, is described Rehman, A., Hussain, M., Farooq, A. \& Akram, M. (2019).

\section{General Properties of Matrix Transformations}

In the classic setting of AHP, pairwise comparison coefficients $\left(a_{i j}\right)$ reflect the chances of alternatives $x_{i}$ and $x_{j}$ becoming a "winner." In this sense $\left(a_{i j}\right)$ - conditional probabilities for the alternative $W\left(x_{i}\right)=\frac{W_{i}}{W_{j}} \times W\left(x_{j}\right)$ of the fact that the alternative $x_{j}$ became the "winner." Ideally, using intermediate real-valued non-negative values of assessments, it is possible to obtain "consistent matrices of pairwise comparisons" (the condition of inverse symmetry and transitivity is kept), by which the alternative that received the maximum weight is determined. The condition of inverse symmetry and transitivity is kept independently of some positive coefficient $\Psi$ (which plays the role of scale), the same for all alternatives $i, j, k \in[1, N]$. If the set of alternatives is closed, then $\Psi$ is determined from the normalization conditions

with interpretation

$$
\sum_{i=1}^{N} W\left(x_{i}\right)=1
$$

$$
\begin{gathered}
a_{i j}=W\left(x_{i}\right) / W\left(x_{j}\right) . \\
W\left(x_{1}\right)=\frac{1}{\sum_{j=1}^{N} a_{j 1}} ; W\left(x_{2}\right)=a_{21} \times W\left(x_{1}\right) ; \ldots ; W\left(x_{N}\right)=a_{N 1} \times W\left(x_{1}\right)
\end{gathered}
$$

For consistent matrices $A=\left(a_{i j}\right)$ ratio

$$
e_{i j}=a_{i j} \times W\left(x_{j}\right) / W\left(x_{i}\right)=1
$$

is carried out for all $i, j \in[1, N]$.

In the case of using AHP in Decision Support System, the purpose of processing the matrices of expert assessments of alternative variants is an alternative with the highest value of $W\left(x_{i}\right)$. The classical AHP assumes that the matrix of pairwise comparisons: complete; each alternative has a nonzero chance to win; it is completely "not agreed" due

International Symposium on the Analytic Hierarchy Process
2

WEB CONFERENCE

DEC. 3 - DEC. 6,2020 
to a partial violation of transitivity. When moving to a linear scale, $W\left(x_{i}\right)$ is converted to $v_{i}=\log \left(W\left(x_{i}\right)\right)$, the matrix of pairwise comparisons $A=\left(a_{i j}\right)$ becomes a skewsymmetric matrix $\bar{A}=\left(\log a_{i j}\right)$ according to the property of inverse symmetry. An analogue of $e_{i j}$ of (3) is the elements of the skew-symmetric error matrix $E_{i j}$ :

$$
E_{i j}=\bar{a}_{i j}-v_{i}+v_{j}
$$

For consistent matrices $E_{i j}=0$ for all $i, j \in[1, N]$. Another indicator of the consistency of skew-symmetric matrices is that for any lines the values $\left(E_{i k}-E_{j k}\right)(k=1, \ldots, N)$ are equal to constant. The essence of the convert of the skew-symmetric matrix $E[t+1]=S_{x}^{i}(E[t])$ with the parameter $x$ is in an elementby-element reduction by $x$ in the $i$-th line and an increase by $x$ in the $i$-th column of the matrix $E[t]$. From the convert properties it follows that at any time $t$, the state of the matrix $E[t]$ is determined by the initial matrix $E\left[t_{0}\right]$ and the accumulation of sums for similar converts, while the order of application of converts of different types is not important.

\section{Indicator That Tracks the Status of Network Connections}

The initial matrix includes two values: $G_{i, j}[t]=1$ when the connection takes place, and $G_{i, j}[t]=0$ when it is absent. For some problems, the connections $G_{i, j}[t]=G_{i, j}$ are stationary, for others it is possible to remove "contradictory" connections in accordance with certain rules.

Choosing bias

$$
\Delta L_{i}[t]=\frac{E_{\max }^{i *}[t]-E_{\max }^{* i}[t]}{2}=\frac{E_{m a x}^{i *}[t]+E_{\min }^{i *}[t]}{2}
$$

where $E_{\max }^{i *}[t]$ is the maximum value of elements of a matrix $E_{i, j}[t], E_{\text {min }}^{i *}[t]$ is the minimum value of elements of a matrix $E_{i, j}[t]$ and $E_{\max }^{* i}[t]$ is the maximum value of elements of a matrix $E_{j, i}[t]$ for which the indicator function $G_{j, i}[t]=1$ (a maximum on column $i$ ) and influencing a matrix $E[t]$ by convert $S_{\Delta L_{i}[t]}^{i}(E[t])$ we have:

$$
\begin{gathered}
E_{i, j}[t+1]=E_{i, j}[t]-\Delta L_{i}[t] \text { where } G_{i j}[t]=1 \\
E_{j, i}[t+1]=E_{j, i}[t]+\Delta L_{i}[t] \text { where } G_{j, i}[t]=1 \\
E_{\text {max }}^{i *}[t+1]=E_{\text {max }}^{* i}[t+1]=\frac{E_{\text {max }}^{i *}[t]+E_{\text {max }}^{* i}[t]}{2} ; E_{\text {min }}^{i *}[t+1]=-E_{\text {max }}^{i *}[t+1]
\end{gathered}
$$

The values $E_{\text {max }}^{i *}[t+1]$ and $E_{\text {max }}^{* i}[t+1]$ are fitted.

We define through the first norm $\|E\|$ the maximum of the matrix $E_{i, j}[t]$ at $G_{i, j}[t]=1$. It follows that if $E_{\max }^{i *}[t]=\|E\|[t]$ is the only maximum at which $E_{\max }^{* i}[t]<\|E\|[t]$, then $\|E\|[t+1]<\|E\|[t]$ (by lines) and if the only maximum is reached on element $E_{i, j}[t]$, then the decrease $\|E\|[t+1]$ is achieved by converting $S_{\Delta L_{j}}^{j}(E[t])$ (by column $j$ ). The second norm $\llbracket E \rrbracket$ is defined therewith as the sum $\llbracket E \rrbracket=\sum_{i=1}^{N}\left|\left(E_{\max }^{i *}+E_{\min }^{i *}\right) / 2\right|$. Due to the proposals made, the process of lowering the first norms is converged. If the first and the second norms are zero, the matrix $E[t]$ becomes zero, and the matrix $\bar{A}$, respectively, 
becomes consistent and is completely determined by the value of the accumulated sums taken with the opposite sign. For uncoordinated matrices $\bar{A}$, the convergence process described in the lemmas results in final states other than the zero matrix $E[t]$. The second norm $\llbracket E \rrbracket$ becomes zero, and the first norm $\|E\|$ becomes equal to the value of $K$, which in the future we will call the consistency criterion of the matrix $\bar{A}$. For a set of lines $I=\{i\}$ for which $E_{\text {max }}^{i *}$ is $K$, in each line $i_{d} \in I$ there is at least one maximum $E_{\text {max }}^{i_{d^{*}}}$ and at least one minimum $E_{\text {min }}^{i_{d^{*}}}$. Other elements of matrix $E$ for which $\left(-K<E_{i_{d} j}<K\right)$ is performed may be temporarily dropped. We create oriented graph $H_{i j}$ from all $\left|E_{i_{d} j}\right|=K$ and consider that the maxima are the entry points to the node $i_{d}$, and the minima are the exit points from $i_{d}$. As a result, $H_{i j}$ will have one or more cycles. Moreover, the resulting graph will not necessarily be connected, the cycles can be inserted in each other.

\section{The Vector Compression Method}

Suppose that there is a table of some data (Table 1).

Table 1. Initial data

\begin{tabular}{l|c|c|c}
\hline $\begin{array}{c}\text { Number of the } \\
\text { line }\end{array}$ & $\begin{array}{c}\text { Maximum value in } \\
\text { the line }\end{array}$ & $\begin{array}{c}\text { Minimum value in } \\
\text { the line }\end{array}$ & $\begin{array}{c}\text { Value of coordinate wise } \\
\text { bias }\end{array}$ \\
\hline 1 & $E_{\max }^{1 *}[t]$ & $E_{\min }^{1 *}[t]$ & $\left(E_{\max }^{1 *}[t]+E_{\min }^{1 *}[t]\right) / 2$ \\
\hline 2 & $E_{\max }^{2 *}[t]$ & $E_{\min }^{2 *}[t]$ & $\left(E_{\max }^{2 *}[t]+E_{\min }^{2 *}[t]\right) / 2$ \\
\hline$\ldots$ & & & \\
\hline $\mathrm{N}$ & $E_{\max }^{N *}[t]$ & $E_{\min }^{N *}[t]$ & $\left.E_{\max }^{N *}[t]+E_{\min }^{N *}[t]\right) / 2$ \\
\hline
\end{tabular}

Let $O_{1}, O_{2}, \ldots, O_{N}$ be a set of comparison objects. Each expert $\exists_{1}, \ni_{2}, \ldots, \ni_{M}$ sets his own logarithmic matrix of pairwise comparisons $\left(\bar{a}_{i j}^{m}\right)$, and an indicator matrix $\left(\bar{G}_{i j}^{m}\right)$. The only condition is that the link graph forms a spanning graph. With the number of pairwise links equal to $(N-1)$ (degenerate case), the spanning graph is a spanning tree. With the number of links equal to $N(N-1) / 2$, a fully connected matrix $\left(\bar{a}_{i j}^{m}\right)$ is formed. The structure of experts, in turn, forms a consistent fully connected spanning graph with paired connections, given in logarithmic notation by the matrix $\left(\bar{\exists}_{i j}^{m}\right)$. Consider a unifying network in the form of a block matrix $\bar{A}$ of dimension $[M \times N, M \times N]$ and a zero vector $v_{i}^{m}[0]$ of dimension $[M \times N]$. Let us calculate local maxima $E_{\text {max }}^{i *}$ and minima $E_{\text {min }}^{i *}$ by lines. Let us recalculate $v_{i}^{m}[t+1]=v_{i}^{m}[t]+\frac{E_{m a x}^{i *}+E_{\min }^{i *}}{3}$, we come back to the beginning of an algorithm, and otherwise if $E_{\text {max }}^{* *}>\varepsilon_{1}$ - we adjust $\bar{A}=\bar{A}-\theta E$ (former $v_{i}^{m}$ can be left) and again we come back to the beginning. Otherwise, the specified accuracy is achieved. We count $v_{i}^{m}$ for each expert and we form for the upper $T_{i}$ and the lower $B_{i}$ assessments of sets of weights $B_{i} \leq v_{i}^{m} \leq T_{i}$. In the matrix elements view, the index consists of a double block index and a double object pair index. It is well to bear in mind that the matrices of the indicator matrix $\left(\bar{G}_{i j}^{m}\right)$ may not match. In this variant the number of free variables is $(M+1) N(N-1) / 2$. With large $N$, it is necessary to aggregate expert data considering the correction of object weights. In this case, the number of free variables will drop to $N(N-1)$ since it will be necessary to introduce upper and lower assessments for elements of one matrix of pairwise comparison. 


\section{Conclusions}

The described approach can be applied to methods of processing data of incomplete examinations, when new connections and new nodes (objects) may appear. This is quite real in practice - the appearance of new objects, which must be quickly tied to the current general picture. For example, in Kohonen neural networks, it is done by introducing a neural gas type into consideration. The proposed method can also be used in a wide range of decision-making tasks, including analysis and quantitative assessment of risks, security management of complex systems and objects, etc.

\section{Key References}

Rubin, D., \& Little, R. (1987) Statistical Analysis with Missing Data. John Wiley \& Sons, Inc., 1987. - 336 p. - ISBN 0-471-80254-9.

Millet, I. (1997) The effectiveness of alternative preference elicitation methods in the analytic hierarchy process, J. Multi-Criteria Decis. Anal., vol. 6, no. 1, pp. 41-51, 1997.

$\mathrm{Xu}, \mathrm{Z}$.S. (2004) Goal programming models for obtaining the priority vector of incomplete fuzzy preference relation. International Journal of Approximate Reasoning, 36:3 (2004) 261-270.

Garcia-Laencina, P.J., Sanco-Gomez, J.-L., \& Figueiras-Vidal, A.R. (2009) Pattern classification with missing data: a review. - London: Springer-Verlag Limited, 2009.

Carmone, F. J., Kara, Jr., A., \& Zanakis, S. H. (1997) A Monte Carlo investigation of incomplete pairwise comparison matrices in AHP, Eur. J. Oper. Res., vol. 102, no. 3, pp. 533-553, Nov. 1997.

Ebenbach, D.H., \& Moore, C.F. (2000) Incomplete information, inferences, and individual differences: The case of environmental judgements, Org. Behav. Human Decis. Process, vol. 81, no. 1, pp. 1-27, Jan. 2000.

Alonso, S., Cabrerizo, F. J., Chiclana, F., Herrera, F., \& Herrera-Viedma, E. (2008) An interactive decision support system based on consistency criteria, J. Mult.-Valued Log. Soft Comput., vol. 14, no. 3-5, pp. 371-386, 2008.

Rehman, A., Hussain, M., Farooq, A., \& Akram, M. (2019). Consensus-Based MultiPerson Decision Making with Incomplete Fuzzy Preference Relations Using Product Transitivity. Mathematics. 7. 185. 